\title{
Half-Metallic Silicon Nanowires: First-Principles Calculations
}

\author{
E. Durgun, ${ }^{1,2}$ D. Çakır, ${ }^{1,2}$ N. Akman, ${ }^{2,3}$ and S. Ciraci ${ }^{1,2, *}$ \\ ${ }^{1}$ Department of Physics, Bilkent University, Ankara 06800, Turkey \\ ${ }^{2}$ UNAM-Institute of Materials Science and Nanotechnology, Bilkent University, Ankara 06800, Turkey \\ ${ }^{3}$ Department of Physics, Mersin University, Mersin, Turkey \\ (Received 28 November 2006; published 21 December 2007)
}

\begin{abstract}
From first-principles calculations, we predict that specific transition metal (TM) atom-adsorbed silicon nanowires have a half-metallic ground state. They are insulators for one spin direction, but show metallic properties for the opposite spin direction. At high coverage of TM atoms, ferromagnetic silicon nanowires become metallic for both spin directions with high magnetic moment and may have also significant spin polarization at the Fermi level. The spin-dependent electronic properties can be engineered by changing the type of adsorbed TM atoms, as well as the diameter of the nanowire. Present results are not only of scientific interest, but also can initiate new research on spintronic applications of silicon nanowires.
\end{abstract}

DOI: 10.1103/PhysRevLett.99.256806

PACS numbers: 73.22. $-\mathrm{f}, 71.15 .-\mathrm{m}, 73.20 . \mathrm{Hb}$

Rodlike, oxidation resistant Si nanowires (SiNW) can now be fabricated at small diameters [1] $(1-7 \mathrm{~nm})$ and display diversity of interesting electronic properties. In particular, the band gap of semiconductor SiNWs varies with their diameters. They can be used in electronic and optical applications [2-4].

In this Letter, we report a novel spin-dependent electronic property of hydrogen terminated silicon nanowires (H-SiNW): when decorated by specific transition metal (TM) atoms they show half-metallic [5,6] (HM) ground state. Namely, due to broken spin degeneracy, energy bands, $E_{n}(\mathbf{k}, \uparrow)$ and $E_{n}(\mathbf{k}, \downarrow)$, split and the nanowire remains to be an insulator for one spin direction of electrons, but becomes a conductor for the opposite spin direction achieving $100 \%$ spin polarization at the Fermi level. Under certain circumstances, depending on the adsorbate and diameter, semiconductor H-SiNWs can also be either a ferromagnetic semiconductor or metal for both spin directions. High-spin polarization at the Fermi level can also be achieved for high TM coverage of specific SiNWs. Present results are of fundamental and technological interest, since room temperature ferromagnetism is already discovered in $\mathrm{Mn}^{+}$-doped SiNW [7]. Once combined with advanced silicon technology, these predicted properties can be realizable making "known silicon" again a potential material with promising nanoscale technological applications in spintronics and magnetism.

Qian et al. [8] have proposed HM heterostructures composed of $\delta$-doped Mn layers in bulk Si. Recently, Son et al. [9] predicted HM properties of graphene nanoribbons. Stable 1D half-metals have been also predicted for TM atom doped armchair single-wall carbon nanotubes [10] and linear carbon chains $[11,12]$, but the synthesis of these nanostructures appears to be difficult.

Our results are obtained from first-principles plane wave calculations [13] (using a basis with maximum kinetic energy of $400 \mathrm{eV}$ ) within generalized gradient approximation (GGA) expressed by PW91 functional [14]. All cal- culations for paramagnetic, ferromagnetic, and antiferromagnetic states are carried out using ultrasoft pseudopotentials [15] and confirmed by using PAW potentials [16]. All atomic positions and lattice constants are optimized by using the conjugate gradient method where total energy and atomic forces are minimized. The convergence for energy is chosen as $10^{-6} \mathrm{eV}$ between two steps, and the maximum force allowed on each atom, is $10^{-3} \mathrm{eV} / \AA$ [17].

Bare $\operatorname{SiNW}(N)$ s (which are oriented along [001] direction and have $N \mathrm{Si}$ atoms in their primitive unit cell) are initially cut from the ideal bulk Si crystal in rodlike forms and subsequently their atomic structures and lattice parameters are relaxed [18]. The optimized atomic structures are shown for $N=21,25$, and 57 in Fig. 1. While bare $\operatorname{SiNW}(21)$ is a semiconductor, bare $\operatorname{SiNW}(25)$ and SiNW(57) are metallic. The average cohesive energy relative to a free $\mathrm{Si}$ atom $\left(\bar{E}_{c}\right)$ is comparable with the calculated cohesive energy of bulk crystal (4.66 eV per $\mathrm{Si}$ atom) and it increases with increasing $N$. The average cohesive energy relative to the bulk $\mathrm{Si}$ crystal, $\bar{E}_{c}^{\prime}$, is small but negative as expected. Upon passivation of dangling bonds with hydrogen atoms all of these SiNWs (specified as H-SiNW) become semiconductor with a band gap $E_{G}$. Because of confinement effect $E_{G}$ was known to increase with decreasing diameter $D$ of $\mathrm{H}-\mathrm{SiNW}$. However, our study reveals that $E_{G}$ depends not only on $D$, but also on the geometry of cross section. In particular, we found that $E_{G}$ of the structure-optimized $\mathrm{H}-\mathrm{SiNWs}$ for a given $N$ depends on whether the bare SiNW is relaxed before it is passivated with $\mathrm{H}$ (as we did here to mimic the growth process) or not [19]. The binding energy of adsorbed hydrogen relative to the free $\mathrm{H}$ atom $\left(E_{b}\right)$ as well as relative to the free $\mathrm{H}_{2}\left(E_{b}^{\prime}\right)$ are both positive and increase with increasing $N$ [19]. Extensive ab initio molecular dynamics calculations have been carried out at $T=500 \mathrm{~K}$ using supercells, which comprise either two or four primitive unit cells of nanowires to lift artificial limitations imposed by periodic boundary condition. After several iterations lasting $1 \mathrm{ps,}$ 
(a)
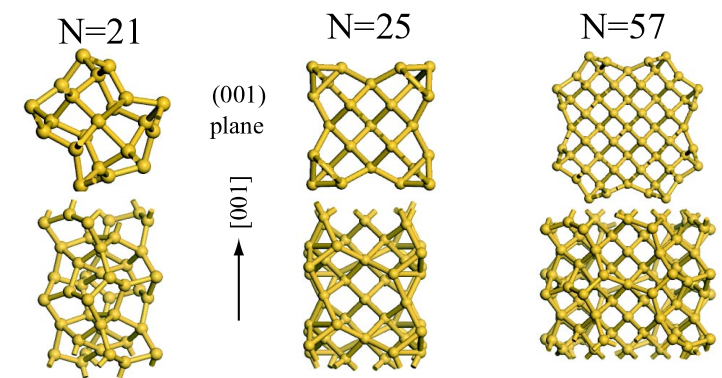

$$
\overline{\mathrm{E}}_{\mathrm{c}}=4.05 \quad \overline{\mathrm{E}}_{\mathrm{c}}=-0.61
$$

$E_{G}=0.6 \mathrm{eV}$

$\overline{\mathrm{E}}_{\mathrm{c}}=4.13 \quad \overline{\mathrm{E}}_{\mathrm{c}}=-0.53$

Metal
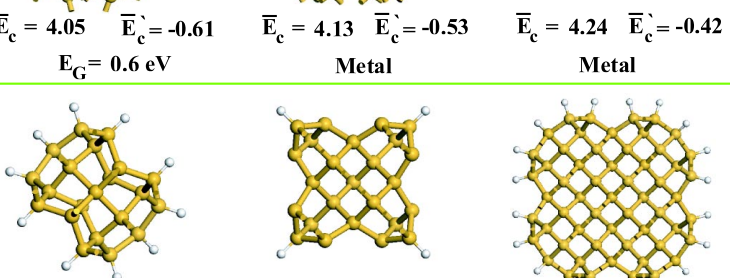

(b)
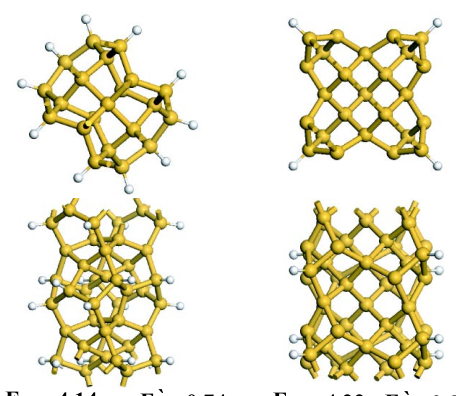

$E_{b}=4.14 \quad E_{b}^{\prime}=0.74$

$E_{G}=0.7 \mathrm{eV}$

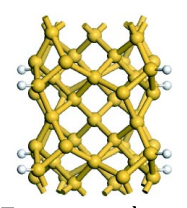

$E_{b}=4.22 \quad E_{b}=0.82$ $E_{G}=1.1 \mathrm{eV}$

(c)
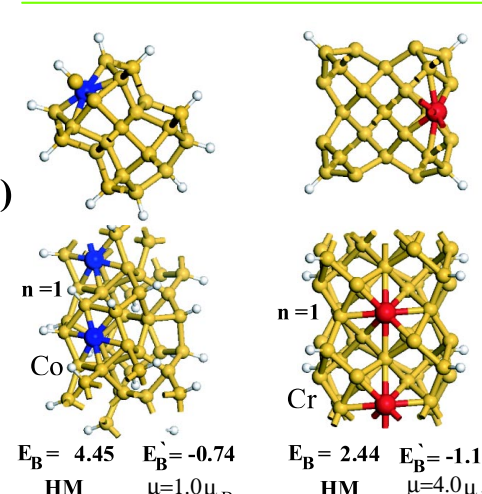

$\begin{array}{cc}E_{B}=2.44 & E_{B}=-1.17 \\ H M & \mu=4.0 \mu\end{array}$
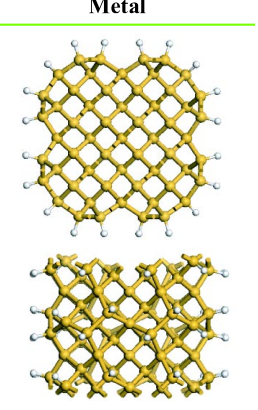

$\mathrm{E}_{\mathrm{b}}=4.28 \quad \mathrm{E}_{\mathrm{b}} \mathrm{i}=\mathbf{0 . 8 8}$ $E_{G}=1.7 \mathrm{eV}$

(d)
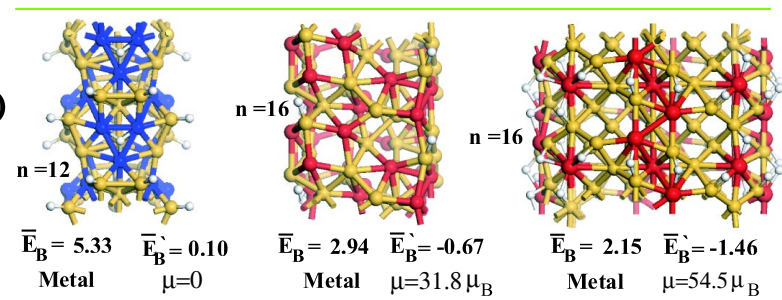

FIG. 1 (color online). Top and side views of optimized atomic structures of various SiNW(N)'s. (a) Bare SiNWs, (b) H-SiNWs, (c) single TM atom adsorbed per primitive cell of $\mathrm{H}-\mathrm{SiNW}(n=$ 1), (d) H-SiNWs covered by $n$ TM atoms. $\bar{E}_{c}, \bar{E}_{c}^{\prime}, E_{b}, E_{b}^{\prime}, E_{G}$, and $\mu$, respectively, denote the average cohesive energy relative to free $\mathrm{Si}$ atom, same relative to the bulk $\mathrm{Si}$, binding energy of hydrogen atom relative to free $\mathrm{H}$ atom, same relative to $\mathrm{H}_{2}$ molecule, energy band gap, and the net magnetic moment per primitive unit cell. Binding energies in regard to the adsorption of TM atoms; i.e., $E_{B}, E_{B}^{\prime}$ for $n=1$ and average values $\bar{E}_{B}, \bar{E}_{B}^{\prime}$ for $n>1$, are defined in the text and in Ref. [22]. Small, largelight, and large-dark balls represent $\mathrm{H}, \mathrm{Si}$, and $\mathrm{TM}$ atoms, respectively. Side views of atomic structure comprise two primitive unit cells of the SiNWs. Binding and cohesive energies are given in $\mathrm{eV} /$ atom. the structure of all $\mathrm{H}-\mathrm{SiNW}(N)$ remained stable. Even though SiNWs are cut from ideal crystal, their optimized structures are reconstructed and hence deviate substantially from crystalline coordination, especially for small diameters. Upon hydrogen termination the structure is healed partly; some peaks in the distribution of interatomic distances coincide with those in the ideal case and approaches the ideal case. The calculated response of the wire under uniaxial tensile strain $\epsilon, \kappa=\partial^{2} E_{T} / \partial \epsilon^{2}$, ranging from 172 to $394 \mathrm{eV} /$ cell, indicates that the strength of $\mathrm{H}-\mathrm{SiNW}(N) \mathrm{s}(N=21-57)$ is rather high.

The adsorption of a single $\mathrm{TM}(\mathrm{TM}=\mathrm{Fe}, \mathrm{Ti}, \mathrm{Co}, \mathrm{Cr}$, and $\mathrm{Mn}$ ) atom per primitive cell, denoted by $n=1$, has been examined for different sites (hollow, top, bridge, etc.) on the surface of $\mathrm{H}-\mathrm{SiNW}(N)$ for $N=21,25$, and 57. In Fig. 1(c) we present only the most energetic adsorption geometry for a specific TM atom for each $N$, which results in a HM state. These are $\mathrm{H}-\mathrm{SiNW}(21)+\mathrm{Co}, \operatorname{SiNW}(25)+$ $\mathrm{Cr}$, and $\operatorname{SiNW}(57)+\mathrm{Cr}$. These nanowires have ferromagnetic ground state, since their energy difference between calculated spin-unpolarized and spin-polarized total energy, i.e., $\Delta E^{m}=E_{T}^{\mathrm{su}}-E_{T}^{\mathrm{sp}}$, is positive. We calculated $\Delta E^{m}=0.04,0.96$, and $0.99 \mathrm{eV}$ for $\mathrm{H}-\mathrm{SiNW}(21)+\mathrm{Co}$, $\mathrm{H}-\mathrm{SiNW}(25)+\mathrm{Cr}$, and $\mathrm{H}-\mathrm{SiNW}(57)+\mathrm{Cr}$, respectively [20]. Moreover, these wires have integer number of unpaired spin in their primitive unit cell. This is in contrast to usually weak binding of TM atoms on single-wall carbon nanotubes which can lead clustering [21]. The binding energy of TM atoms $\left(E_{B}\right)$ on $\mathrm{H}-\mathrm{SiNW}$ s is high and involves significant charge transfer from TM atom to the wire [22]. Mulliken analysis indicates that the charge transfer from Co to $\mathrm{H}-\mathrm{SiNW}(21)$ is 0.5 electrons. The charge transfer from $\mathrm{Cr}$ to $\mathrm{H}-\mathrm{SiNW}(25)$ and $\mathrm{H}-\mathrm{SiNW}(57)$ is even higher ( 0.8 and 0.9 electrons, respectively). Binding energies of adsorbed TM atoms relative to their bulk crystals $\left(E_{B}^{\prime}\right)$ are negative and hence indicate endothermic reaction. Because of very low vapor pressure of many metals, it is probably better to use some metal precursor to synthesize the structures predicted here.

The band structures of HM nanowires are presented in Fig. 2. Once a Co atom is adsorbed above the center of a hexagon on the surface of H-SiNW(21) the spin degeneracy is split and the whole system becomes magnetic with a magnetic moment of $\mu=1 \mu_{B}$ (Bohr magneton per primitive unit cell). Electronic energy bands become asymmetric for different spins: bands of majority spins continue to be semiconducting with relatively smaller direct gap of $E_{G}=0.4 \mathrm{eV}$. In contrast, two bands of minority spins, which cross the Fermi level, become metallic. These metallic bands are composed of Co- $3 d$ and $\mathrm{Si}-3 p$ hybridized states. The density of majority and minority spin states, namely $D(E, \uparrow)$ and $D(E, \downarrow)$, display a $100 \%$ spin polarization $P=\left[D\left(E_{F}, \uparrow\right)-D\left(E_{F}, \downarrow\right)\right] /\left[D\left(E_{F}, \uparrow\right)+D\left(E_{F}, \downarrow\right)\right]$ at the Fermi level, $E_{F}$. H-SiNW(25) $+\mathrm{Cr}$ is also HM. The indirect gap of majority spin bands has reduced to $0.5 \mathrm{eV}$. 

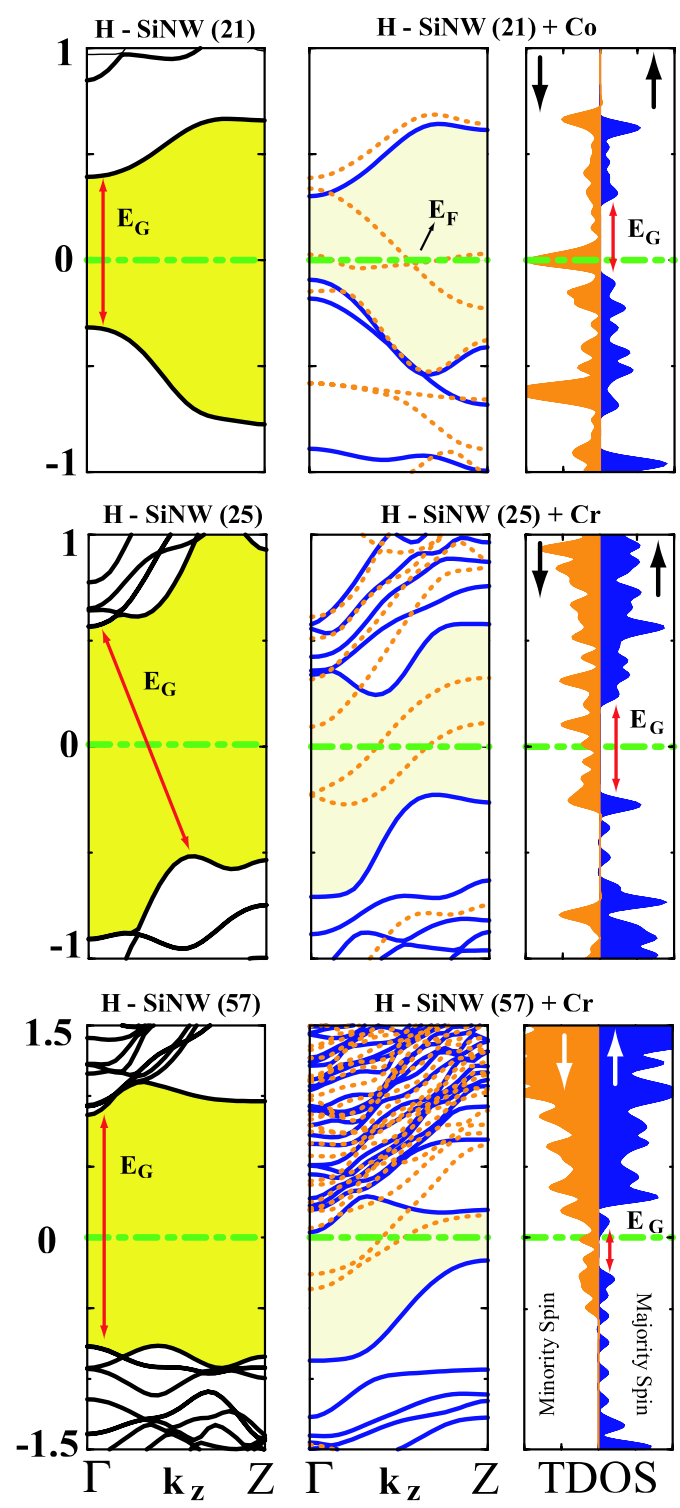

FIG. 2 (color online). Band structure and spin-dependent total density of states (TDOS) for $N=21,25$, and 57. Left panels: semiconducting $\mathrm{H}-\mathrm{SiNW}(N)$. Middle panels: half-metallic $\mathrm{H}-\mathrm{SiNW}(N)+\mathrm{TM}$. Right panels: density of majority and minority spin states of $\mathrm{H}-\mathrm{SiNW}(N)+\mathrm{TM}$. Bands described by continuous and dotted lines are majority and minority bands. Zero of energy is set to $E_{F}$.

On the other hand, two bands constructed from Cr- $3 d$ and Si-3p hybridized states cross the Fermi level and hence attribute metallicity to the minority spin bands. Similarly, $\mathrm{H}-\mathrm{SiNW}(57)+\mathrm{Cr}$ is also HM. The large direct band gap of undoped $\mathrm{H}-\mathrm{SiNW}(57)$ is modified to be indirect and is reduced to $0.9 \mathrm{eV}$ for majority spin bands. The minimum of the unoccupied conduction band occurs above but close to the Fermi level. The net magnetic moment is $4 \mu_{B}$. Using the energy difference of antiferromagnetic and ferromagnetic states calculated with PAW potential, we present a rough estimate of Curie temperature of half-metallic
$\mathrm{H}-\mathrm{SiNW}+\mathrm{TMs}$ as $T_{C} \simeq 10,290$, and $700 \mathrm{~K}$ for $N=21$, 25 , and 57 , respectively.

We note that DFT underestimates the band gap of HSiNW [23]. In the case of HM, H-SiNW + TM presented in Fig. 2, the lowest conduction band and highest valence band are reminiscent of those of $\mathrm{H}-\mathrm{SiNW}$; the shrunk band gap still attributes a semiconducting behavior for majority spins. As for the metallic minority spin bands in the gap, they are reminiscent of the linear TM chain having the same lattice parameter as H-SiNW + TM, except that their dispersions increase due to indirect TM-TM coupling through $\mathrm{Si}$ atoms. Under uniaxial compressive strain the minimum of the conduction band of majority spin states rises above the Fermi level. Conversely, it becomes semimetallic under uniaxial tensile strain. Since (majority spin) conduction and valence bands of both $\mathrm{H}-\mathrm{SiNW}(21)+\mathrm{Co}$ and $\mathrm{H}-\mathrm{SiNW}(25)+\mathrm{Cr}$ are away from $E_{F}$, their $\mathrm{HM}$ behavior is robust under uniaxial strain. The form of two metallic bands crossing the Fermi level eliminates the possibility of Peierls distortion. Nevertheless, HM ground state of SiNWs is not common to all adsorbed TM atoms. For example, $\mathrm{H}-\mathrm{SiNW}(N)+\mathrm{Fe}$ is a consistently ferromagnetic semiconductor with different $E_{G, \uparrow}$ and $E_{G, \downarrow}$. $\mathrm{H}-\mathrm{SiNW}(N)+\mathrm{Mn}(\mathrm{Cr})$ can be either ferromagnetic metal or HM depending on $N$.

To see whether spin-dependent GGA properly represents localized $d$ electrons or whether possible on-site repulsive Coulomb interaction destroys the HM, we also carried out LDA $+U$ calculations [24]. We found that insulating and metallic bands of opposite spins coexist up to high values of repulsive energy $(U=4)$ for $N=25$. For $N=$ 57, HM persists until $U \sim 1$. Clearly, the HM character of $\mathrm{H}-\mathrm{SiNW}+\mathrm{Cr}$ revealed in Fig. 2 is robust and unique behavior.

Finally, we note that HM state predicted in TMdecorated $\mathrm{H}$-SiNWs occurs in perfect infinite structures; complete spin polarization may deviate slightly from $P=$ $100 \%$ due to the finite extent of devices. Even if the exact $\mathrm{HM}$ character corresponding to $n=1$ is disturbed for $n>$ 1, the possibility that some $\mathrm{H}-\mathrm{SiNWs}$ having high-spin polarization at $E_{F}$ at high TM coverage can be relevant for spintronic applications. We therefore investigated electronic and magnetic structures of H-SiNW + TM for $n>1$ as described in Fig. 1(d).

$\mathrm{H}-\mathrm{SiNW}(25)$ is ferromagnetic for different levels of $\mathrm{Cr}$ coverage and has a high net magnetic moment. For example, $n=8$ can be achieved by two different geometries; both geometries are ferromagnetic with $\mu=19.6$ and $32.4 \mu_{B}$ and are metallic for both spin directions. Interestingly, while $P$ is negligible for the former geometry, the latter one has $P=0.91$ and hence is suitable for spintronic applications (see Fig. 3). Similarly, Cr covered H-SiNW(57) with $n=8$ and 16 are both ferromagnetic with $\mu=34.3(P=53)$ and $\mu=54.5 \mu_{B}(P=0.33)$, respectively. The latter nanostructure having magnetic mo- 


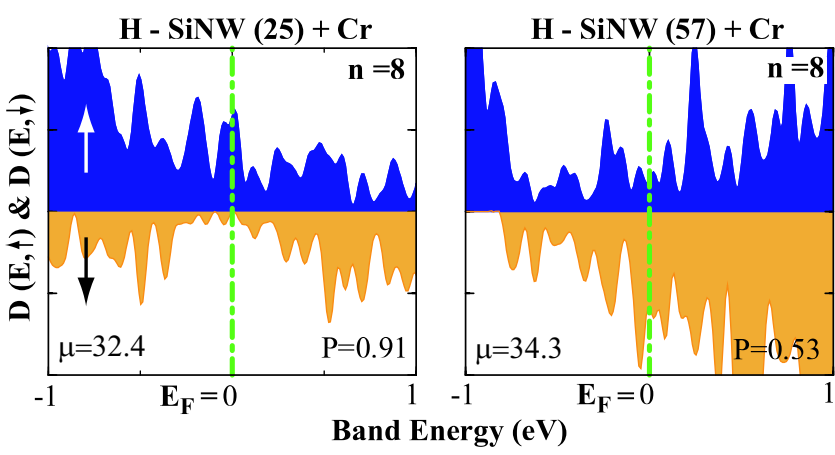

FIG. 3 (color online). $\quad D(E, \downarrow)$ density of minority (light) and $D(E, \uparrow)$ majority (dark) spin states. (a) $\mathrm{H}-\mathrm{SiNw}(25)+\mathrm{Cr}, n=8$; (b) $\mathrm{H}-\mathrm{SiNW}(57)+\mathrm{Cr}, n=8 . P$ and $\mu$ indicate spin polarization and net magnetic moment (in Bohr magnetons per primitive unit cell), respectively.

ment as high as $54.5 \mu_{B}$ can be a potential nanomagnet. Clearly, not only total magnetic moment, but also the spin polarization at $E_{F}$ of TM covered H-SiNMs exhibits interesting variations depending on $n, N$, and the type of TM.

In conclusion, hydrogen passivated SiNWs can exhibit half-metallic state when decorated with specific TM atoms. Resulting electronic and magnetic properties depend on the type of adsorbed TM atom, as well as on the diameter of the nanowire. When covered with more TM atoms, the perfect half-metallic state of $\mathrm{H}-\mathrm{SiNW}$ is disturbed, but for certain cases, the spin polarization at $E_{F}$ continues to be high. High magnetic moment obtained at high TM coverage is another remarkable result which may lead to the fabrication of nanomagnets for various applications. Briefly, functionalizing silicon nanowires with TM atoms presents us a wide range of interesting properties, such as half-metals, 1D ferromagnetic semiconductors, or metals and nanomagnets. We believe that our findings hold promise for the use of silicon-unique material of microelectronics - in nanospintronics including magnetoresistance, spin-valve, and nonvolatile memories.

*ciraci@fen.bilkent.edu.tr

[1] D. D. D. Ma et al., Science 299, 1874 (2003).

[2] Y. Cui et al., Nano Lett. 3, 149 (2003).

[3] Y. Huang, X.F. Duan, and C. M. Lieber, Small 1, 142 (2005).

[4] X. F. Duan et al., Nature (London) 421, 241 (2003).

[5] R. A. de Groot et al., Phys. Rev. Lett. 50, 2024 (1983).

[6] W. E. Pickett and J. S. Moodera, Phys. Today 54, No. 5, 39 (2001).

[7] W. H. Wu et al., Appl. Phys. Lett. 90, 043121 (2007).

[8] M. C. Qian et al., Phys. Rev. Lett. 96, 027211 (2006).
[9] Y.-W. Son, M. L. Cohen, and S. G. Louie, Nature (London) 444, 347 (2006); Phys. Rev. Lett. 97, 216803 (2006).

[10] C.-K. Yang, J. Zhao, and J.P. Lu, Nano Lett. 4, 561 (2004); Y. Yagi et al., Phys. Rev. B 69, 075414 (2004).

[11] S. Dag et al., Phys. Rev. B 72, 155444 (2005).

[12] E. Durgun et al., Europhys. Lett. 73, 642 (2006).

[13] We used vasP: G. Kresse and J. Hafner, Phys. Rev. B 47, R558 (1993). Charge transfer, orbital hybridization, and local magnetic moments have been obtained from SIESTA code using local basis set, P. Ordejon, E. Artacho, and J. M. Soler, Phys. Rev. B 53, R10441 (1996).

[14] J. P. Perdew et al., Phys. Rev. B 46, 6671 (1992).

[15] D. Vanderbilt, Phys. Rev. B 41, R7892 (1990).

[16] P. E. Blochl, Phys. Rev. B 50, 17953 (1994).

[17] All structures have been treated within supercell geometry using the periodic boundary conditions with lattice constants of $a$ and $b$ ranging from $20 \AA$ to $25 \AA$ depending on the diameter of the $\mathrm{SiNW}$ and $c=c_{o}$ ( $c_{o}$ being the optimized lattice constant of SiNW along the wire axis). Convergence tests with respect to kinetic energy cutoff and number of $k$-point sampling have been performed. In the self-consistent potential and total energy calculations the Brillouin zone is sampled in the $\mathbf{k}$ space within Monkhorst-Pack scheme [H.J. Monkhorst and J.D. Pack, Phys. Rev. B 13, 5188 (1976)] by $(1 \times 1 \times 21)$ mesh points. Calculations have been carried out also for supercells with $c=2 c_{o}$ and $c=4 c_{o}$ to allow freedom for structural reconstruction and magnetic state.

[18] Numerous theoretical studies on SiNW have been published in recent years. See, for example: A. K. Singh et al., Nano Lett. 6, 920 (2006); Q. Wang et al., Phys. Rev. Lett. 95, 167202 (2005); Q. Wang, Nano Lett. 5, 1587 (2005).

[19] $E_{G}(N)$ of H-SiNW's is calculated to be 2.5 and $2.1 \mathrm{eV}$ for $N=21$ and 37, respectively, if the underlying $\mathrm{SiNW}$ is not relaxed before $\mathrm{H}$ passivation. For the same $\mathrm{H}$-SiNWs $E_{b}$ and $E_{b}^{\prime}$ are, respectively, 3.93 and $0.53 \mathrm{eV}$ for $N=21$, 4.08 and $0.68 \mathrm{eV}$ for $N=37$.

[20] Spin-polarized calculations have been carried out by starting with different initial $\mu$ values and subsequently by relaxing them. Whether antiferromagnetic ground state exists in H-SiNW $(N)$ + TM's has been explored by doubling the size of supercell.

[21] E. Durgun et al., Phys. Rev. B 67, 201401(R) (2003); J. Phys. Chem. B 108, 575 (2004).

[22] Binding energy corresponding to $n=1$ is calculated by the following expression: $E_{B}=E_{T}[\mathrm{H}-\operatorname{SiNW}(N)]+$ $E_{T}[\mathrm{TM}]-E_{T}[\mathrm{H}-\mathrm{SiNW}(N)+\mathrm{TM}]$ in terms of the total energy of optimized $\mathrm{H}-\operatorname{SiNW}(N)$ and $\mathrm{H}-\operatorname{SiNW}(N)+\mathrm{TM}$ and the total energy of the string of TM atoms having the same lattice parameter $c_{o}$ as $\mathrm{H}-\operatorname{SiNW}(N)+\mathrm{TM}$, all calculated in the same supercell. Hence $E_{B}$ can be taken as the binding energy of single isolated TM atom, since the coupling among adsorbed TM atoms has been subtracted. For $n>1, \bar{E}_{B}(\mathrm{TM})$ is taken as the free TM atom energy, and hence $\bar{E}_{B}^{\prime}$ includes the coupling between TM atoms. For this reason $\bar{E}_{B}^{\prime}>0$ for $\mathrm{H}-\operatorname{SiNW}(21)+\mathrm{Co}$ at $n=12$.

[23] X. Zhao et al., Phys. Rev. Lett. 92, 236805 (2004).

[24] S. L. Dudarev et al., Phys. Rev. B 57, 1505 (1998). 